\title{
Application of evidential reasoning approach in enterprise performance evaluation system
}

\author{
Tiantian BAO, Ruijia ZHAO, Yue YU \\ Transportation Management College \\ Dalian Maritime University \\ Dalian, China
}

\begin{abstract}
Enterprise performance evaluation is a comprehensive evaluation problem with uncertainty. An integrated evaluation index system of enterprise performance is constructed by contrasting and analyzing economic value added, key performance index, balanced score card and other methods. Furthermore, a mathematical model is put forward to solve enterprise performance evaluation problem based on evidential reasoning approach. Then an enterprise performance evaluation system, which is designed through analyzing evaluation process, is realized with multi-language programming technology. Finally, the application analysis verifies the rationality of the method and the stability of evaluation system.
\end{abstract}

Keywords-comprehensive evaluation; evidential reasoning; evaluation system; enterprise performance evaluation

\section{INTRODUCTION}

In most industries, performance evaluation shows the management ability of a company in a certain operating period, which has great effects on producing and operating activities, and is critical for improving enterprise competitiveness. An objective, fair and precise comprehensive evaluation result can be obtained based on a reasonable evaluation index system and an effective evaluation approach[1]. For example, Shen constructed the performance evaluation index system by using the analytical hierarchy process [2]. Fu presented the method based on evidential reasoning algorithm [3]. Lei applied analytic hierarchy process (AHP) to supplier performance evaluation [4]. Due to enterprise performance involves all aspects of the enterprise operation, enterprise performance evaluation cannot be considered from the single financial aspect, but should contain multiple factors. Therefore, an integrated evaluation index system of enterprise performance is established through the contrastive analysis of economic value added (EVA), key performance index (KPI), balanced score card (BSC), etc. Then a mathematics model is put forward based on evidential reasoning approach (ERA) to solve the evaluation problems with uncertainty which include both qualitative and quantitative attributes in practice. Evidential reasoning approach was presented and improved by Yang and $\mathrm{Xu}[5,6]$ to implement the decision analysis of the hybrid multi-attributes decision making with uncertainty. In recent years, this method has been widely used in maritime risk assessment, ship type evaluation, competitiveness comparison, etc.[7-12]But the manual calculation task is heavy for decision maker due to the high complexity of ERA. In order to assist corporate investors and operators to make investment and management decisions, an enterprise performance evaluation system should be designed.

\section{ENTERPRISE PERFORMANCE EVALUATION MODEL}

\section{A. Evaluation index system}

With the change of the enterprise pattern of organization and management, besides traditional financial assessment, there are some other methods of enterprise assessment coming into being, representatively, such as EVA, KPI, BSC, etc. Among those, EVA is one of the estimate methods for the company economic profit, which represents the balance between net operating profit after taxes and the capital cost. In addition, KPI is a performance measurement to determine a few indexes which have closer relationship with the strategic goals. Furthermore, BSC is a complete set of systematic performance evaluating indicators. Based on strategic theory and core competence theory, it is made up of four dimensions including financial, customer, internal business process and learning and growth. In China, Assets Supervision and Administration Commission of the State Council (SASAC) promulgated the relevant rules of Enterprise Performance Evaluation.

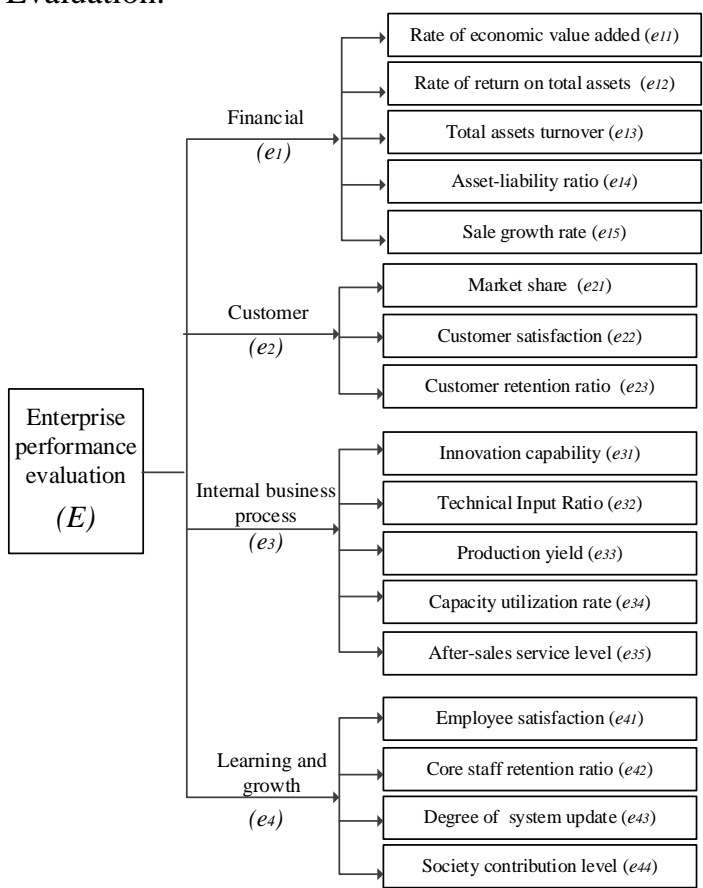

Fig. 1. The integrated evaluation index system of enterprise performance

By analyzing the above achievements, it can be seen that EVA and the relevant rules in China excessively emphasize financial indexes, while KPI and BSC just provide a theoretical conception and framework. Therefore, an 
integrated evaluation index system of enterprise performance is built in a hierarchical structure including four dimensions of BSC (see Fig.1). And the criteriaof this index system is identified by the combination of EVA, KPI and the relevant rules in China.

\section{B. Mathematical model}

1) Suppose there is a simple index system with two levels, which contains a total attribute $y$ and some basic attributes $e_{i}(i=1,2, \cdots L)$. The weight of $e_{i}$ is denoted by $\omega_{i}$, satisfying $0 \leq \omega_{i} \leq 1$ and $\sum_{i=1}^{L} \omega_{i}=1 \cdot e_{i}$ can be evaluated by $\mathrm{N}$ different grades, denoted by $H=\left\{H_{1}, H_{2}, \cdots H_{n}, \cdots H_{N}\right\}$ and $H_{n} \succ H_{n+1}$, in which $H_{n} \succ H_{n+1}$ represents $H_{n}$ is better than $H_{n+1}$. But for quantitative attributes, the best and worst values are obtained easily, denoted by $\left[H_{\text {best }}, H_{\text {worst }}\right]$, which can be transformed into the evaluation grades with $\mathrm{N}$ levels as follows.

$$
H_{n}=H_{\text {best }}-\frac{\left(H_{\text {best }}-H_{\text {worst }}\right)(n-1)}{N-1}, n=1, \cdots, N
$$

2) The initial evaluation set of $e_{i}$ can be stated by $S\left(e_{i}\right)=\left\{\left(H_{n}, \beta_{n, i}\right), n=1, \cdots, N\right\}$, where $\beta_{n, i}$ is represented as the belief degree of the alternative which belongs to grade $H_{n}$ on attribute $e_{i}$.For qualitative basic criteria, the belief degree can be extracted as (2), which is given as the single level evaluation by experts.

$$
\beta_{n, i}=q_{n, i} / Q_{i} \quad n=, \cdots, N
$$

where $q_{n, i}$ represents the number of experts who agree that attribute $e_{i}$ belongs to grade $H_{n}, Q_{i}$ represents the total number of experts.

For quantitative basic criteria, the belief degree is calculated by (3), in which $h$ is determined by investigating and collecting the relevant data.

$$
\beta_{n, i}=\frac{h-H_{n+1, i}}{H_{n, i}-H_{n+1, i}}, \beta_{n+1, i}=1-\beta_{n, i}
$$

where $H_{n, i} \leq h \leq H_{n+1, i}$.

3) According to evidence combination rule, the evaluation set is denoted as $S(y)=\left\{\left(H_{n}, \beta_{n}\right), n=1, \cdots, N\right\} \cdot \beta_{n}$ represents the belief degree of the total attribute $y$ which belongs to grade $H_{n}$, and can be gained as follows:

$$
\begin{gathered}
m_{n, i}=\omega_{i} \beta_{n, i} \\
m_{H, i}=1-\sum_{n=1}^{N} m_{n, i}
\end{gathered}
$$

Let $\bar{m}_{n, i}=1-\omega_{i}$ and $\tilde{m}_{n, i}=\omega_{i} \beta_{H, i}$, then $\bar{m}_{H, i j}+\tilde{m}_{H, i j}=m_{H, i j}$.

$m_{n, I(i+1)}=K_{I(i+1)}\left[m_{n, I(i)} m_{n, i+1}+m_{H, I(i)} m_{n, i+1}+m_{n, I(i)} m_{H, i+1}\right]$

$\tilde{m}_{H, I(i+1)}=K_{I(i+1)}\left[\tilde{m}_{H, I(i)} \tilde{m}_{H, i+1}+\bar{m}_{H, I(i)} \tilde{m}_{H, i+1}+\tilde{m}_{H, I(i)} \bar{m}_{H, i+1}\right]$

$$
\begin{aligned}
\bar{m}_{H, I(i+1)} & =K_{I(i+1)} \bar{m}_{H, I(i)} \bar{m}_{H, i+1} \\
m_{H, I(i)} & =\widetilde{m}_{H, I(i)}+\bar{m}_{H, I(i)}
\end{aligned}
$$

where $K_{I(i+1)}=\left[1-\sum_{t=1}^{N} \sum_{j=1, j \neq t}^{N} m_{t, I(i)} m_{j, i+1}\right]^{-1}$ is a normalization factor,. Then $S(y)$ can be computed by(10).

$$
\beta_{n}=\frac{m_{n, I(L)}}{1-\bar{m}_{H, I(L)}}, \beta_{H, i}=\frac{\widetilde{m}_{H, I(L)}}{1-\bar{m}_{H, I(L)}}
$$

4) For $M$ alternatives $a_{j}(j=1,2, \ldots, M)$, let $u\left(H_{n}\right)$ be the utility function of $H_{n}$.And $u(y)$ represents the utility value of alternative $a_{j}$ on total attribute $y$, and can be calculated by (11). Then the ranking and optimization of alternatives can be gained through comparing the utility values.

$$
u(y)=\sum_{n=1}^{N} \beta_{n} u\left(H_{n}\right) \text { for } a_{j}
$$

\section{THE PROCESSES AND DESIGN OF EVALUATION SYSTEM}

\section{A. Process analysis of evaluation system}

According to the evaluation model above, the processes of evaluation system are analyzed (see Fig.2). Firstly, the decision makers build the evaluation index system (see Fig.1) and prepare the corresponding information of attributes and grades. Secondly, alternatives are selected and described in detail. Thirdly, the corresponding initial values are sorted out. If the numbers of grades between the index and sub-index are different, the mapping relationship should be determined. Finally, the comprehensive evaluation values of all alternatives are given invarious forms by integrating the initial information.

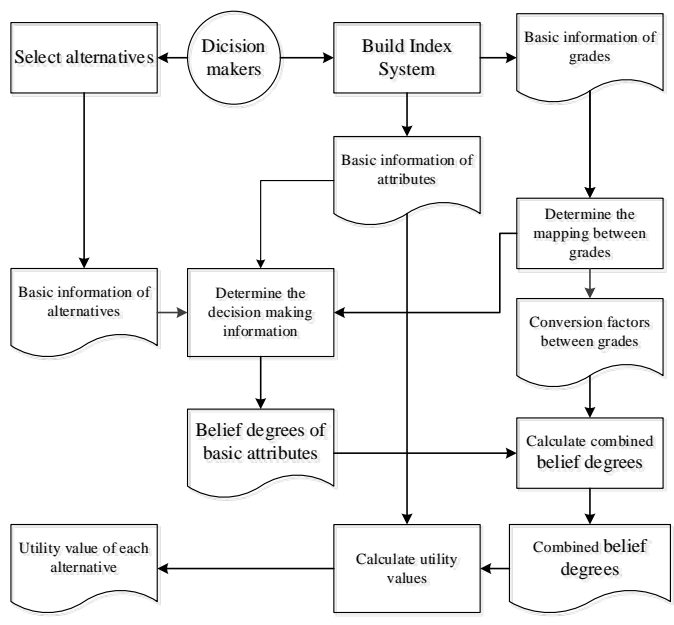

Fig.2 Transaction flow diagram of system

\section{B. Function design of evaluation system}

According to process analysis above, the evaluation system is divided into three main function modules, as shown in Fig.3.

(1) Index system management module

This module is used to manage the evaluation index system of enterprise performance, in which the index system can be created, opened or deleted in view of the actual situation. 


\section{(2) Data management module}

This module, as the core of the evaluation system, is divided into 4 sub-modules. 1) Attributes management sub-module: it is used to save, update and delete the information of all attributes and show the attributes relationship in a tree structure; 2) Grades management sub-module: it is used to save, update and delete the grades information for attributes. For quantitative attributes, the conversion calculation in (1) is achieved. For qualitative attributes, the system acquiescently provides the mapping transformation. 3) Alternatives management sub-module: This sub-module is used to save, update and delete the information of alternatives.4) Evidential reasoning sub-module: it is used to obtain the comprehensive evaluation results by integrating the initial evaluation information according to (2-11).

\section{(3) Evaluation and analysis module}

This module is used to analyze comprehensive evaluation information through multi-angle based on bar graph and list forms. 1) Ranking sub-module is used to show the ranking order of alternatives based on the utility values. 2) Belief degree sub-module is used to express evaluation results in the distributed way according to the values of belief degrees. 3) Overall comparison sub-module is used to describe the comprehensive evaluation information of the main criteria (both total attributes and primary attributes). 4) Report sub-module is used to save the evaluation results as Excel files.

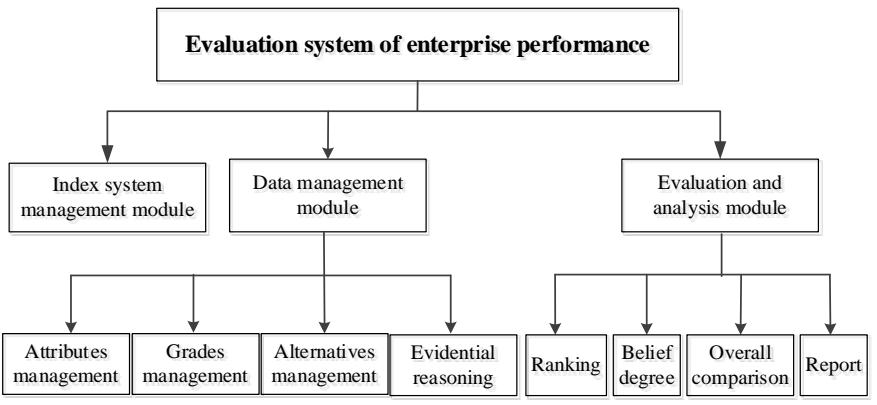

Fig.3 The function design of system

\section{Multi-language programming of evaluation system}

In order to obtain the required data conveniently, timely and accurately, the database of evaluation system is built by Microsoft Office Access to organize, store, maintain and retrieve a large amount of data required in system evaluation. The application platform is realized by the GUI system and Visual components of the Visual Basic, which includes every main function module and human-computer interaction interface. The mixed programming with two programming languages can ensure complete function, clear structure and being easy to use[13].

\section{APPLICATION EXAMPLES FOR EVALUATION SYSTEM}

In order to verify the rationality of evaluation model and the stability of evaluation system, the water transportation industry in China is regarded as the application example. Suppose that there are three shipping enterprises, named as company A, company B and company C. The weight is determined by using AHP according to experts' opinions. The weights of primary attributes $(e 1 \ldots e 4)$ is $\mathrm{W}=\{0.3,0.25,0.25,0.2\}$. And the weights of other attributes and the initial evaluation information are shown in Table I. Then using the enterprise performance evaluation system, the evaluation model is established, as shown in Figure 4. And the evaluation results of each shipping company are given by evaluation calculation, as shown inFig.5- Fig.7.

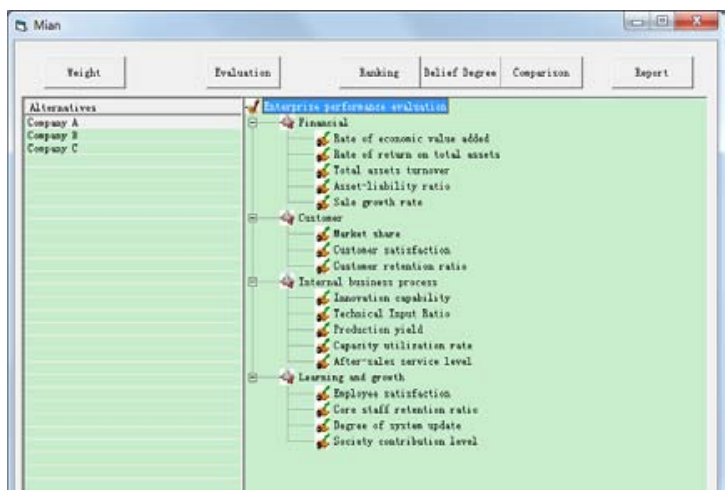

Fig.4 Evaluation model of enterprise performance

TABLE I. INITIAL EVALUATION INFORMATION OF EACH SHIPPING ENTERPRISES

\begin{tabular}{|c|c|c|c|c|c|}
\hline \multirow{2}{*}{ Attributes } & \multirow{2}{*}{ Weights } & \multirow{2}{*}{ Grades set } & \multicolumn{3}{|c|}{ Initial values } \\
\hline & & & company A & company $B$ & company $C$ \\
\hline$e_{11}$ & 0.24 & $(5,2.3,-2,-4.7,-11.4)$ & 2.0 & 4.5 & 1.4 \\
\hline$e_{12}$ & 0.22 & $(4.5,2.2,-1.0,-2.1,-8.6)$ & 1.7 & 3.9 & 1.1 \\
\hline$e_{13}$ & 0.18 & $(0.8,0.6,0.5,0.2,0.1)$ & 0.5 & 0.7 & 0.65 \\
\hline$e_{14}$ & 0.18 & $(46.3,60.6,70,87.3,99.8)$ & 65 & 51.6 & 60 \\
\hline$e_{15}$ & 0.18 & $(14.8,8.7,3,-4.7,-12)$ & 9.5 & 11 & 8.2 \\
\hline$e_{21}$ & 0.4 & {$[1,0]$} & 0.3 & 0.2 & 0.2 \\
\hline$e_{22}$ & 0.3 & (best, good, average, poor, worst) & $(0.5,0.5,0,0,0)$ & $(0.4,0.5,0,0,0)$ & $(0.6,0.2,0,0,0)$ \\
\hline$e_{23}$ & 0.3 & {$[1,0]$} & 0.75 & 0.7 & 0.7 \\
\hline$e_{31}$ & 0.25 & (best, good, average, poor, worst) & $(0.5,0.3,0.2,0,0)$ & $(0.5,0.45,0,0,0)$ & $(0.7,0.3,0,0,0)$ \\
\hline$e_{32}$ & 0.25 & $(0.7,0.6,0.5,0.4,0.3)$ & 0.5 & 0.6 & 0.65 \\
\hline$e_{33}$ & 0.2 & {$[1,0]$} & 0.9 & 0.85 & 0.95 \\
\hline$e_{34}$ & 0.15 & {$[1,0]$} & 0.7 & 0.8 & 0.75 \\
\hline$e_{35}$ & 0.15 & (best, good, average, poor, worst) & $(0.8,0,0,0,0)$ & $(0.5,0.5,0,0,0)$ & $(0.6,0.2,0,0,0)$ \\
\hline$e_{41}$ & 0.3 & (best, good, average, poor, worst) & $(0.8,0,0,0,0)$ & $(0.5,0.4,0,0,0)$ & $(0.5,0.5,0,0,0)$ \\
\hline$e_{42}$ & 0.2 & {$[1,0]$} & 0.85 & 0.75 & 0.8 \\
\hline$e_{43}$ & 0.2 & (best, good, average, poor, worst) & $(0.5,0.5,0,0,0)$ & $(0,1,0,0,0)$ & $(0.5,0.3,0,0,0)$ \\
\hline$e_{44}$ & 0.3 & (best, good, average, poor, worst) & $(0,1,0,0,0)$ & $(0.4,0.6,0,0,0)$ & $(0.3,0.6,0,0,0)$ \\
\hline
\end{tabular}




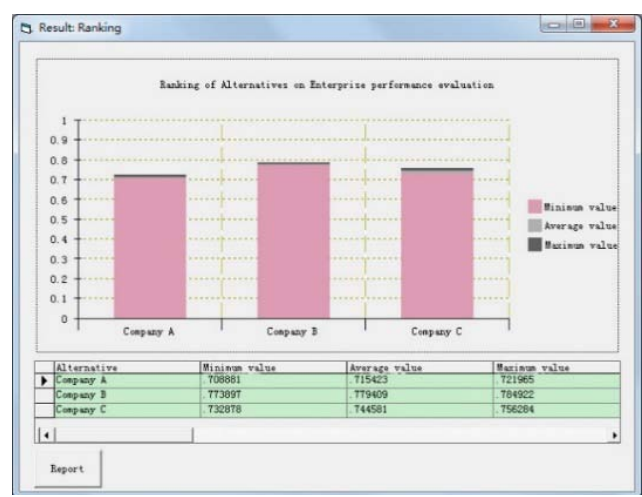

Fig.5 Ranking of alternatives on enterprise performance evaluation

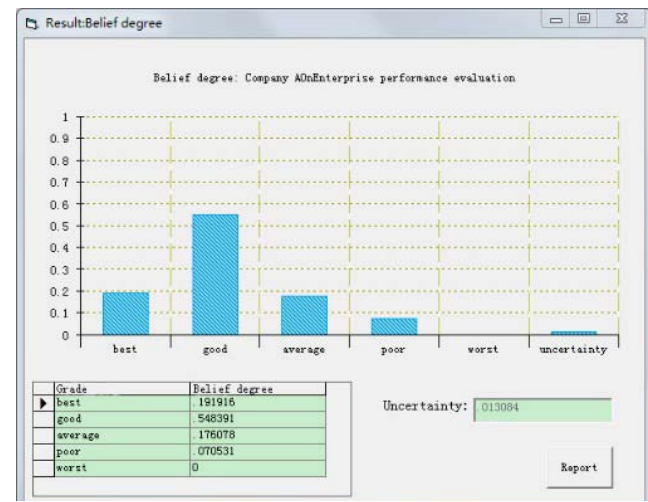

Fig.6 Belief degree of company A on enterprise performance evaluation

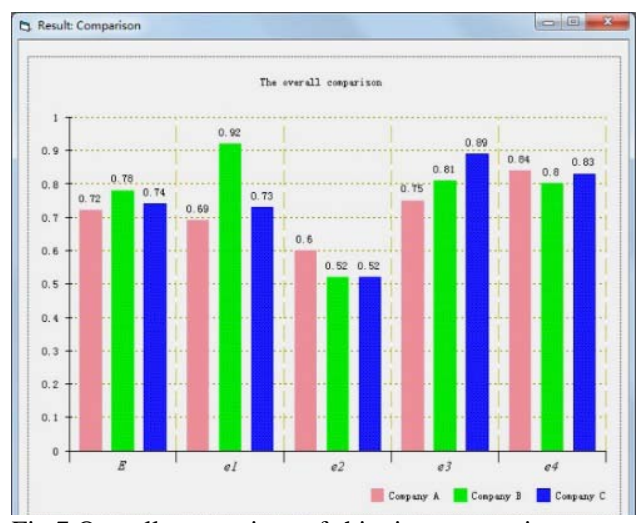

Fig.7 Overall comparison of shipping companies

As seen from Fig.5, Company B has the highest utility value that is 0.78 ; Company $\mathrm{C}$ has the lower utility value that is 0.75; Company A has the lowest utility value that is 0.72 . For company A (see Fig.6), the highest value of belief degree is 0.55 denoted as "good" followed by the "best" of 0.19 . In addition, the overall evaluation results of all three shipping companies exist some uncertainty characterized by the grey part in Figure 5, because some basic attributes have uncertainty in the evaluation process (see Table I). For example, the attributes of "level of after-sale service" and "employee satisfaction" both have 0.2 degree of uncertainty, which leads to 0.01 degree of uncertainty in comprehensive performance evaluation of company A. As is concluded in Fig.7, Company A has some advantages in customer and obvious disadvantages in finance; company B has prominent advantages in finance and can keep balance in other dimensions; company $\mathrm{C}$ has advantages in internal operation process and learning and growing relatively.

\section{CONCLUSION}

According to the characteristics of the enterprise performance evaluation, an enterprise performance evaluation model is put forward in combination with EVA, KPI and BSC based on evidential reasoning approach. Due to the high complexity of evaluation calculation, an evaluation system of enterprise performance is designed and developed by hybrid programming technology. This system can improve the evaluation efficiency and enhance the speed and precision of data processing. Finally, three shipping companies are taken as example for the empirical analysis that proves the rationality of evaluation model and the stability of evaluation system. This system can provide reference and support for comprehensive evaluation of enterprise performance.

\section{REFERENCES}

[1] Li D, Zeng F R. A Summarizing on the enterprise performance evaluation methods[J]. Communication of Finance and Accounting, 2011, (1):49-50.

[2] Shen Z D. Analysis of Analytic Hierarchy Process to Build a State-owned Enterprise Evaluation System[J]. Auditing Research,2013,(2):106-112.

[3] Fu Y H, Zhang huaguang, Tang Jiafu. Performance Evaluation for Selection of Supplier and Decision-Making Based on Evidential Reasoning [J]. Journal of Northeastern University (Natural Science), 2009,11:1546-1549.

[4] Lei X H, You X Y, Evaluation of Outsourcing service providers' performance on analytic hierarchy process to support decision-making [J]. Journal of TONGJI University (Natural Science), 2014, 11: 1770-1775

[5] Yang J B. Rule and utility based evidential reasoning approach for multiple attribute decision analysis under uncertainty[J]. European Journal of Operational Research,2001,131: 31-61.

[6] Yang J B, Xu D L. On evidential reasoning algorithms for multiple attribute decision analysis under uncertainty[J]. IEEE TRANS. SYST., MAN, CYBERN.,2002, 32:289-304.

[7] Wang J, Sii H S, Yang J B. Use of advances in technology for maritime risk assessment[J]. Risk Analysis,2004,24(4):1041-1063.

[8] Xie X L, Xu D L, Yang J B, et al. Ship selection using a multiple-criteria synthesis approach[J]. Journal of Marine Science and Technology, 2008,13(1):50-62.

[9] Xie X L, Yang J B, Xu D L, et al. Uncertaintyand preference modeling for multiple criteria vehicle evaluation[J]. International Journal of Computational Intelligence Systems,2010,3(6):688-708.

[10] Shen R G, Xie X L. Choosing the place of mother port of cruiseship using MADM under uncertainty [J]. Journal of Dalian Maritime University, 2007,33(1):71-75

[11] Chen J, Xie X L. Comparative Analysis on Competitiveness of Asia-Europe Continent Bridge based on Evidence Organum [J]. RAILWAY TRANSPORT AND ECONOMY,2012,34(11):82-87.

[12] Yang J B, Xu D L, Xie X, et al. Multicriteria evidential reasoning decision modeling and analysis-prioritizing voices of customer[J].Journal of the Operational Research Society,2011,62(9):1638-1654.

[13] Chen W W Decision support system and development [M].Beijing: Tsinghua University Press, 2014. 